\title{
An Integrated Wind and Hydro Power System Using Switched Reluctance Generator
}

\author{
Ibrahim Al-Bahadly \\ School of Engineering and Advanced Technology, Massey University, Palmerston North, New Zealand \\ Email: i.h.albahadly@massey.ac.nz
}

How to cite this paper: Al-Bahadly, I. (2018) An Integrated Wind and Hydro Power System Using Switched Reluctance Generator. Journal of Power and Energy Engineering, 6, 1-19. https://doi.org/10.4236/jpee.2018.62001

Received: January 11, 2018

Accepted: February 6, 2018

Published: February 9, 2018

Copyright (C) 2018 by author and Scientific Research Publishing Inc. This work is licensed under the Creative Commons Attribution International License (CC BY 4.0).

http://creativecommons.org/licenses/by/4.0/ (c) (i) Open Access

\begin{abstract}
This research work seeks to make renewable energy more reliable, cost effective, and accessible by exploring a different energy combination system to that currently applied to wind and hydro power. Instead of the usual electrical combination of wind and hydro generators, this work involved combining a water and wind turbine mechanically, before driving an electrical generator. This new combination system was modeled and optimized in MATLAB, using a direct combination system commonly found in multi-engine helicopters. The system was found to operate satisfactorily, however it is mechanically more complex than current electrical combining systems. Research was undertaken regarding wind and water resource availability, and the turbines were chosen with these taken into consideration. Various combination systems were explored, including torque and speed split mechanical combinations, conventional electrical combination, and using a modified switched reluctance generator as a method of electro-mechanical combination. The generator selected for this work is a three phase 12/8 Switched Reluctance (SR) machine. A detailed winding polarity having four poles per phase and their effect on the performance of the machine is observed.
\end{abstract}

\section{Keywords}

Wind Energy, Hydro Turbine, Switched Reluctance Generator

\section{Introduction}

In traditional hybrid energy system energy obtained from different renewable energy sources is collected at a common DC bus [1] [2] [3] [4]. Alternatively, these sources are connected electrically through power electronics interface to extract maximum energy from the sources and for optimal use of harvested energy [5] [6] [7]. In contrast to the traditional system of connecting renewable energy sources, 
this work involves designing and modelling a renewable electricity generation system whereby a small wind turbine and small water turbine are mechanically combined to drive a single generator [8] [9] [10] [11].

All renewable energy applications are very dependent on site geography and resource availability. The available resources were researched and settled before choosing turbines and generators. Following that, various methods of combination are researched and discussed. The chosen system is then implemented using computer simulation software, and the output from the simulation is used to compare with existing systems of combination. A cost estimate as installed is provided, and a CAD mockup of a suitable physical system design is presented.

Currently, the application of the Switched Reluctance (SR) machine as a generator concentrates more on high speed applications such as for the aircraft industries [12]. To date, the operation of the machine in the low and medium speed range is still under study.

The study on SR generator focuses on the feasibility of the machine for wind energy applications [13]. One of the main criterions for the wind energy application is on maximizing the amount of energy captured during the low speed range. Various proposed control methods for SRG are targeted for high speed applications whereby the objectives were confined to maximize the torque production, minimize losses and also to minimize torque ripple [14] [15].

There are not many literatures which focus on the low and medium speed operation. The current chopping mode control method in generating operation is normally used at low speed for wind energy conversion system (WECS) application [16]. A power control strategy was used to maintain the required load demand. Another method to control the output power is achieved by using a fuzzy logic controller to regulate the turn on angle whilst the turn off angle is kept constant [17]. The resources on the application of the machine for the control strategies in wind energy are still limited. The method based on constant output power requires the machine to follow the set of reference power. This limits the potential of the machine to extract energy according to the wind velocity. Hence this work proposes the procedure to maximize the generated power by employing the heuristic method during a wide range of speed.

The use of renewable energy technologies, both large and small scale, is largely dependent on the geography in the area of application. In order to simplify development, the project is directed towards a small scale implementation. Typically this will involve a rural property or lifestyle block, where the land owner has access to a source of flowing water, and steady wind. Based on an average household power consumption of $8000 \mathrm{kWh}$ per year, this system will aim to generate approximately $1 \mathrm{~kW}$ on average.

There is, however, argument for considerably less than that, because with some care and attention to energy efficiency power usage could realistically be half of that. Also considered when choosing components is the desired lifetime of the system. For the purposes of this study that will be taken as 10 years. A 
factor of safety of at least 10 was applied when selecting or designing components.

Small scale renewable energy applications are inherently specific to the site they are implemented on. This makes it very difficult to research and design a system that can be generally applied. For this reason a suitable site has been assumed, with a source of hydro energy and wind energy in close proximity. Examples of such locations in New Zealand include valley floors facing into the prevailing wind (typically westerly), and mountain ranges such as the Ruahine and Tararua Ranges. Exposed coastal areas with streams could also suit. The specific parameters assumed for this study are:

- Water flow: 20 litres/sec, 5 metres head.

- Wind: $5-6 \mathrm{~m} / \mathrm{s}$ on average, feasible to place a small wind turbine in clean air.

The wind speed was chosen based on information from NIWA [18]. Figure 1 illustrates the available wind resource for the whole of New Zealand. The water supply is deliberately a small estimate to maximize the applicability of this project to a wider selection of sites.

\section{Wind Turbine Selection}

The Background choice of a wind turbine was between three possibilities, see Table 1.

The model given in [19] was analyzed and the following equation giving output torque as a function of shaft speed $\omega$, was produced. Note that this works with normalized speeds and torques, i.e. Expects $\omega$ in the range of 0 - 1 (1 being

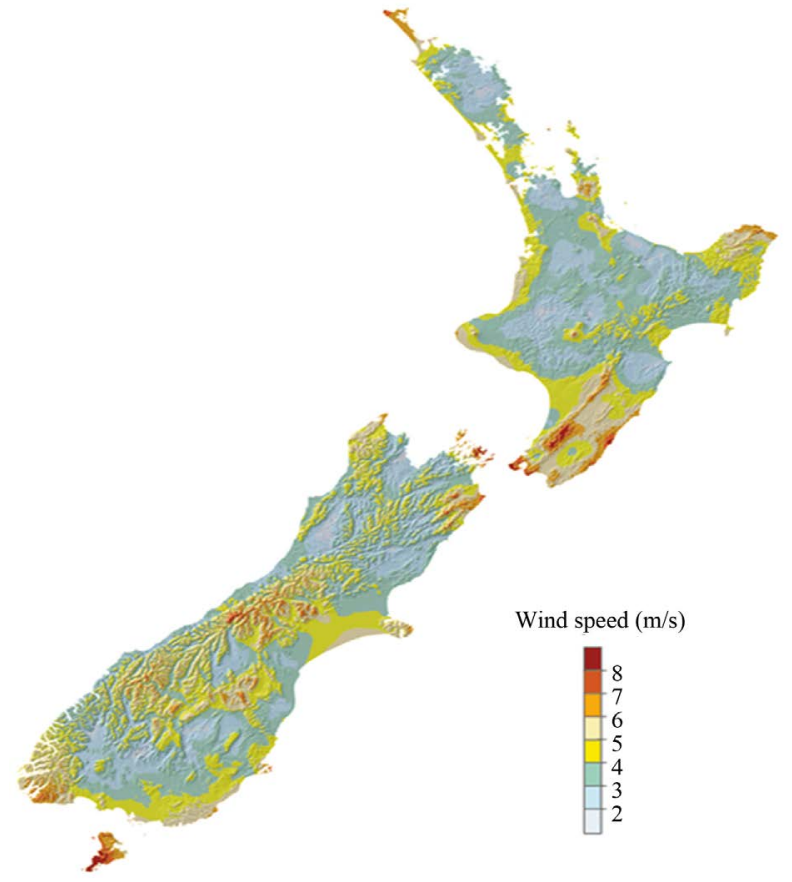

Figure 1. New Zealand wind resource. 
Table 1. Wind turbine design matrix.

\begin{tabular}{|c|c|c|c|c|c|c|c|}
\hline & 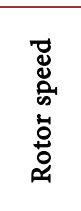 & 苛 & 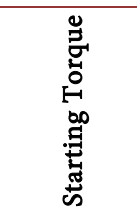 & 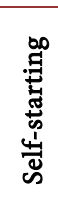 & 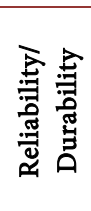 & 预 & 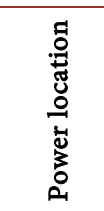 \\
\hline Three blade HAWT & High & Low & Low & Yes & Fair & High & Top \\
\hline American wind mill & Low & High & Very High & Yes & Good & Fair & Top \\
\hline VAWT & Low & Mid & Low & No & Poor & Fair & Ground \\
\hline
\end{tabular}

the rated wind velocity for the turbine), and gives the output torque in the range $0-\sim 1$ (1.011 being the stall torque, which is clearly shown in Equation (2)).

$$
\text { Torque }=0.0608 \omega^{3}+2.1288 \omega^{2}+0.5264 \omega+1.011 .
$$

With this equation in hand, the overall model for the wind turbine required for the project was produced. It can be broken down as shown in Figure 2.

\section{Hydro Turbine Selection}

Several different types of water turbines and wheels were considered, see Table 2 for a comparison between these turbines. The specified pressure head of 5 meters ruled out undershot and crossflow, and made overshot undesirable because of the physical size. Therefore Turgo type of turbine as shown in Figure 3 was thought to be suitable. Turgos have the advantage of being self-cleaning, due to there being no recirculating water flows inside the turbine housing. The water jet enters the turbine runner from above and exits below, so the used water does not interfere with the incoming jet. They are fairly easy to construct, and relatively cheap. Another significant factor in the choice of water turbine was the availability of mathematical model of the turbine that could be implemented in MTALAB and incorporated into main combination model. Large scale models for both Pelton and Turgo were available however they did not scale well to the very small scale that this work is studying. The discovery of a small scale Turgo model was a contributing factor in the decision. The model used was an implementation of a model found in [20] with the findings validated experimentally, and the described model calculates turgo speed/torque characteristics from first principles, using conservation of momentum. This model can be broken into process flow as shown in Figure 4.

The turbine parameters are particularly important to this model because it calculates from first principles.

\section{Method of Coupling}

The method of coupling both the renewable energy sources is the most significant part of this work. The possibilities are as follows:

1) Electrical combining.

2) Mechanical. 


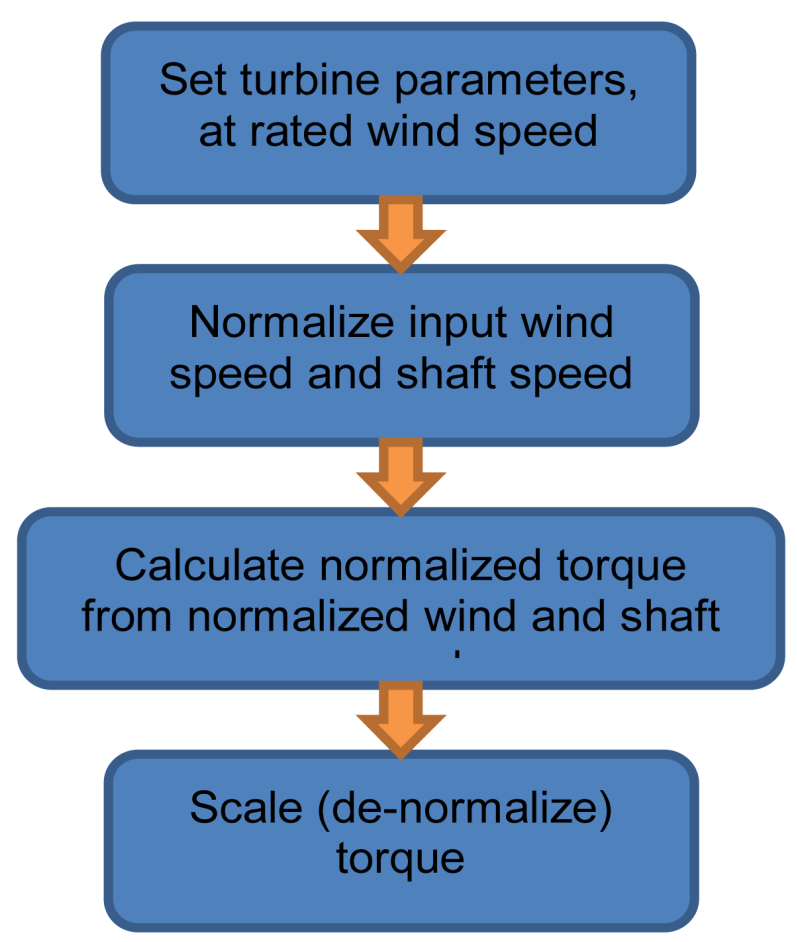

Figure 2. Wind turbine model.

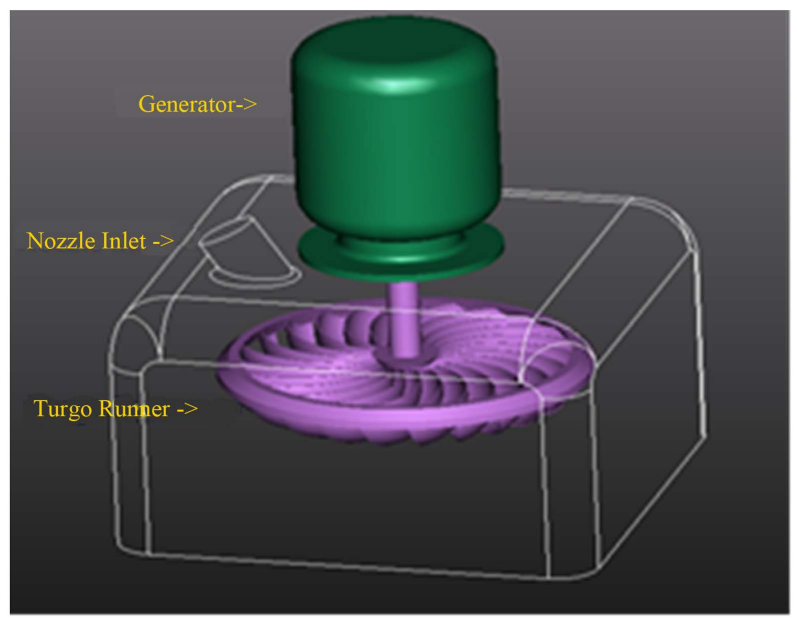

Figure 3. Turgo turbine.

Table 2. Hydro turbine design matrix.

\begin{tabular}{ccccc}
\hline & & & & \\
\hline Overshot & Small & Medium to high & Pico to small & Good \\
Undershot & Very small & Medium to high & Pico to micro & Good \\
Pelton & Medium to high & Low to medium & Micro to large & Good \\
Turgo & Small to high & Low to medium & Pico to large & Good \\
Crossflow & Small to medium & High & Micro to medium & Good \\
\hline
\end{tabular}




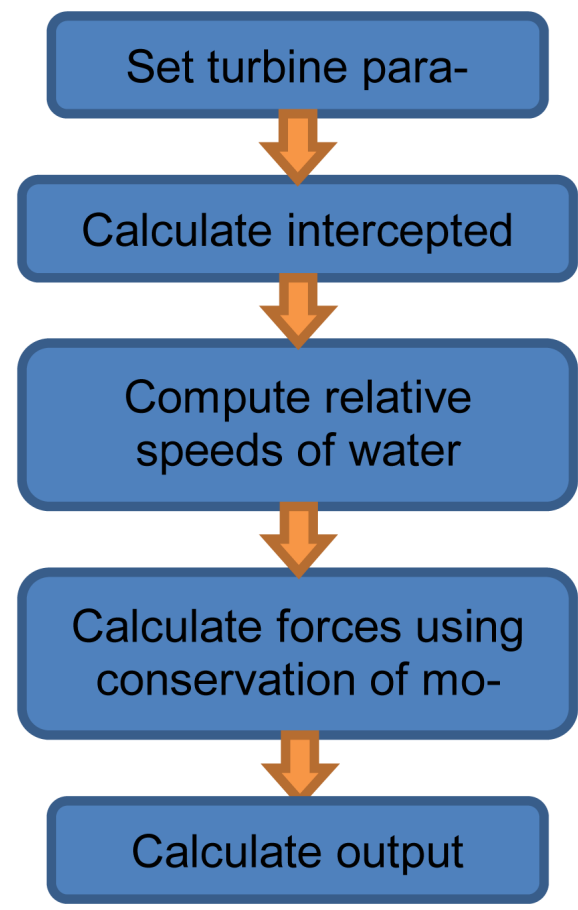

Figure 4. Turgo model.

a) Constant torque ("speed-split").

b) Constant speed ("torque-split").

3) Electromechanical.

Electrical combination as shown in Figure 5 is the current norm. In this method, a wind turbine is installed separately to the hydro turbine, using separate electrical generators, and their outputs are combined in the electrical system of the installation. The two systems are mechanically independent.

Electromechanical combination is a way of combining the two turbine outputs, and would involve a generator that had two rotors, instead of a rotor and stator. In this case, the two rotors would rotate in opposing directions, against each other. Because they rotate against each other and no torque is applied across the generator housing, the input torques must be equal but opposite. For reasons discussed below, this could be problematic.

If the outputs were to be combined in a purely mechanical fashion, then driving a conventional generator, two variations are possible. These are constant torque, and constant speed. Constant torque means the two inputs must have equal torques, but the input speeds can vary with respect to each other and the frame of reference. Two methods of obtaining this were considered, using a differential gearbox, and a planetary gearbox. Both of these examples are used in the automotive industry.

A differential gearbox shown in Figure 6 will be immediately familiar to anyone with a working knowledge of automobiles, as they are universally used to supply the engine power to the wheels on either side, and provide an ability to accommodate a speed differential between the driven wheels (required when 


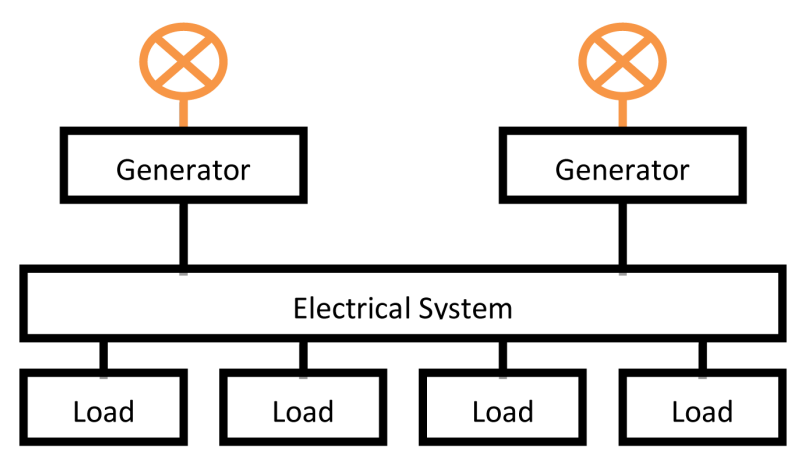

Figure 5. Conventional electrical combination.

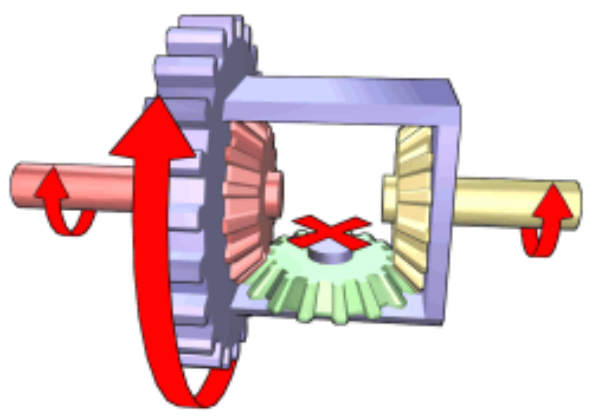

Figure 6. Differential gearbox.

turning). While in this case the differential has a single input and two outputs, the system works fine the other way. This is evident when your car is up on jacks, and you rotate one wheel, causing the opposite wheel to turn the other way.

A planetary gearbox is commonly used in electric drills to reduce the motor speed to a usable speed for drilling, and also in some aircraft to reduce the engine speed to one usable for the propeller or rotors. Toyota also uses planetary gearboxes to combine the petrol engine, two electric motor/generators, and wheels in the Toyota Hybrid System [21]. This system acts as both a continuously variable transmission for the petrol engine and electric motors, and as a way of allowing any combination of power flow between the motor/generators, petrol engine, and wheels. While this system works well in practice, it needs high level supervisory control to coordinate the various power sources and loads, and is quite complex The main disadvantage to a constant torque method like this is caused by the fact that the speeds of the inputs can vary with respect to each other, but the torques will be constant. If the system has inertia, which any physical system will, then the input speeds may be unstable, particularly if the speed-torque curve of a driving source is flat. This phenomenon is known as hunting, as the power sources tend to "hunt" around the operating point and never settle.

A second disadvantage is that if one source is driving the load, the other's starting torque will be equal to the driving source (depending on relative gearing ratios, it may be different, but certainly non-zero). The system could be made to 
allow torque differentials also, but such arrangements generally involve friction (and thus power loss), and a complex mechanism to achieve it. Constant speed means the inputs must have the same speed in order to contribute to the output. Their torque's add together in the output, and may vary with respect to each other. In this case, two or more turbine engines drive the main rotor at a set speed. This arrangement makes it possible for one engine to fail, leaving the remaining operative engine(s) to continue powering the aircraft. A freewheel clutch on each input to the combining gearbox prevents the failed engine from being driven by the operative ones, which could be dangerous depending on how the engine has failed, not to mention the waste of power.

In its most basic form, this gearbox is very simple, with two input gears that both drive a single output gear. Of course extra reduction may be added if appropriate for the application. The main drawback to this method is that the inputs must have the same speed relative to each other. Also a free wheel clutch is required to prevent power loss to a malfunctioning or slow input. The advantages include zero starting torque (indeed an input will not be loaded until it reaches the speed that the system is currently operating at), and perhaps most significantly, simplicity. Generally speaking, the simpler a system, the more reliable it will be, or it will be easier to make reliable, than a more complex system.

Using the chosen wind turbine, the output shaft must be put through a 90 degree gearbox in order to pass vertically down to ground level where the hydro turbine and combining gearbox is. As it is expected that the hydro and wind turbines are offset laterally, a total of three 90 degree gearboxes are required, which can incorporate the necessary gear ratios. Figure 7 illustrates the proposed physical system design.

\section{Generator Selection}

The generator selected for this work is a three phase 12/8 Switched Reluctance (SR) machine as depicted in Figure 8. A machine with an odd number of phases produce symmetrical flux path between the phases, which result in high machine performance. However, the study was limited to machines having only two poles

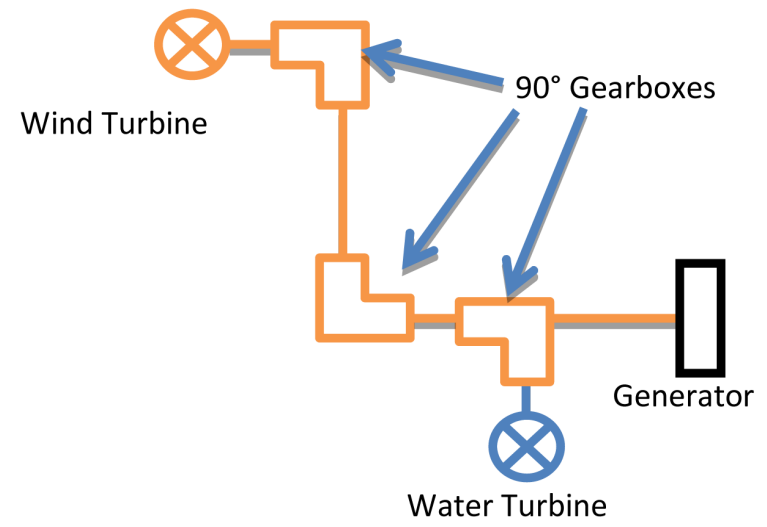

Figure 7. Physical design for the proposed system. 


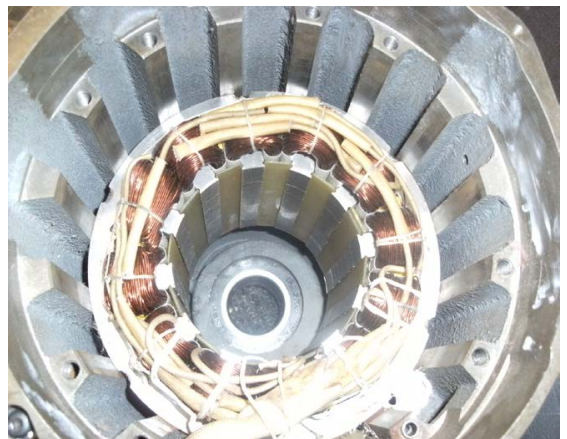

(a)

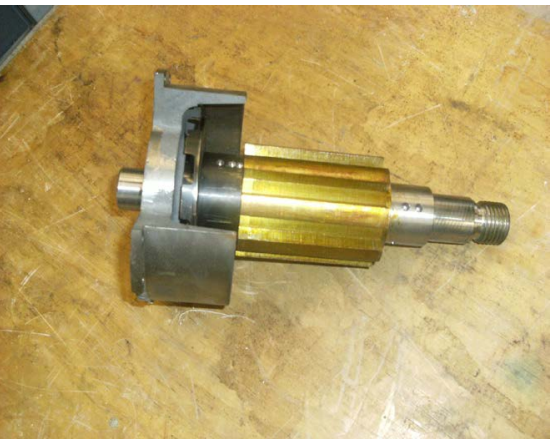

(b)

Figure 8. Configuration of a 3 phase 12/8 machine under study (a) stator (b) rotor.

per phase. A detailed winding polarity having four poles per phase and their effect on the performance of the machine is observed. The study involves a single four poles per phase and simultaneous two phase excitation with the Single phase excitation: NSNS and NSSN winding configuration.

Based on the observation under the single pulse excitation, the NSNS configuration gives the best choice of winding polarity for a three phase four poles machine. A comparison between the magnetization curves of the flux linkage versus current strengthens the choice of this configuration as depicted in Figure 9. The curve is obtained based on the flux created by the magnetomotive force (MMF) applied to the phase winding, which is represented by the following equation:

$$
H=\frac{N i}{l}=\frac{\mathrm{MMF}}{l}
$$

where $H$ is the magnetizing intensity, $N$ is the number of turns, $l$ is the length of the core and $i$ the phase current. The magnetizing intensity is directly proportional to the MMF. The applied MMF produces flux, $\lambda$ which increases as current is increased. Up to a certain point, an increase in the MMF produces no change in the flux. At this point, the material is said to be saturated. The NSSN winding configuration has the smallest area between the aligned and unaligned curve due to the flux leakage whereas the NSNS winding configuration has the largest curve.

The second aspect on maximizing the percentage of generated power is to identify the control parameters affecting the generated power. The SR machine stores the electric energy in the form of magnetic field in the air gap during the excitation stage then release the stored energy together with the mechanical energy during the generation stage. Hence, the operation of the machine can be categorized into two sections; excitation and generation. Therefore, the optimal performance of the machine can be determined according to its generation and excitation stage during each operating cycle.

The control variables affecting both the excitation and generation stage is identified and analysed based on the voltage across the phase winding. The variable 


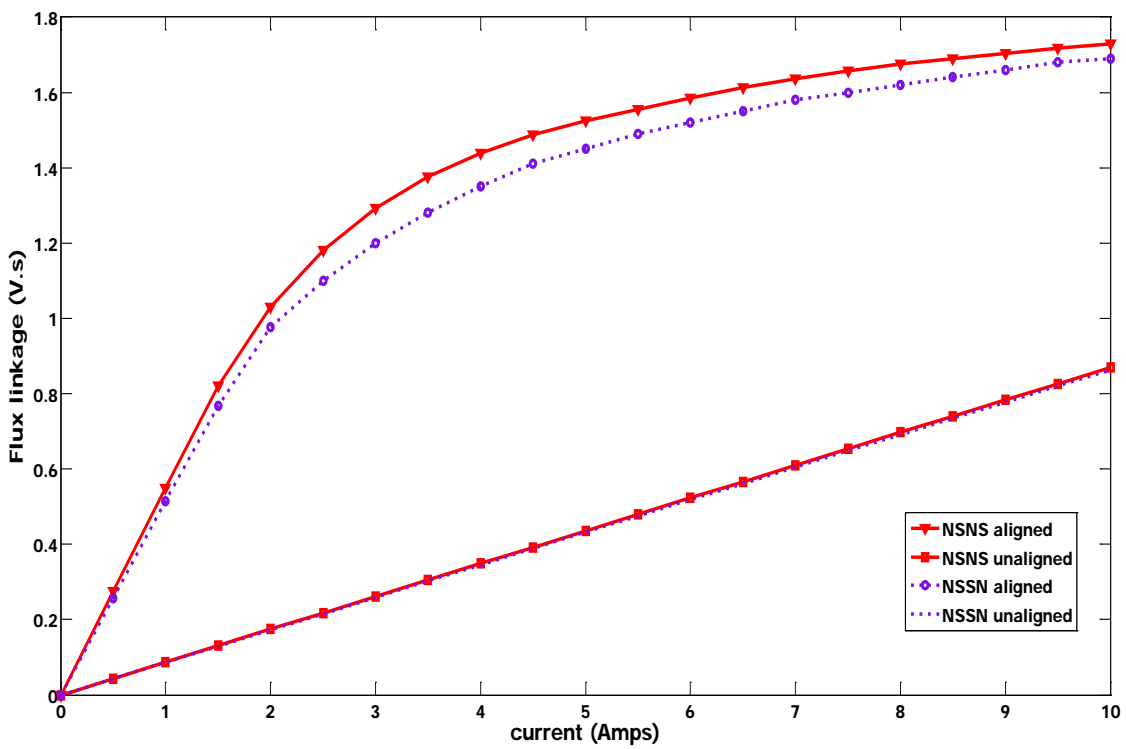

Figure 9. Comparison of magnetization curve for various winding polarity for a three phase 12/8 SR machine.

which provides the highest percentage of generated power over the excitation power is grouped as the optimal variables. Since the machine is highly nonlinear, it is not easy to determine its optimal variables. Hence the heuristic method is employed to obtain an accurate profile of the variables.

From the collected data, the optimal firing angles that yield the highest percentage of generated power at each different speed and voltage level are identified. The effect of increasing the excitation voltage on the shape of phase current for a certain value of firing angle is shown in Figure 10. This finding is crucial in developing the control scheme that can maximize the generating operation of the machine. Based on this analysis, single pulse mode is selected to operate the machine during the low speed range as opposed to the conventional current chopping mode. The optimal angles are grouped according to the optimal voltage level at each speed range.

\section{Modeling}

With a rated power of $1 \mathrm{~kW}$, and designed for wind energy applications, it seemed a good fit for this application. The datasheet supplied by the manufacturer contained all the information needed to model the generator in Simulink. The graphs as shown in Figure 11 were plotted in an Excel spreadsheet, and a polynomial fit was applied to the points to obtain a system equation relating rotation speed to power, voltage, and torque.

Power generated was given by the equation

$$
P(\omega)=-0.0032 \omega^{3}+0.6836 \omega^{2}-1.52769 \omega .
$$

The torque applied on the shaft by the generator (of course opposing the current motion) is given by 


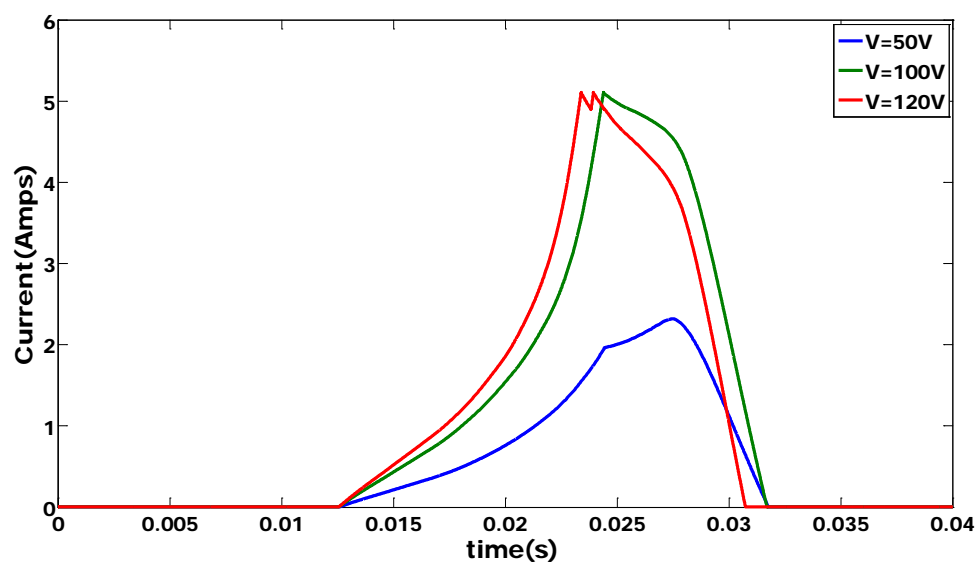

Figure 10. Effect of terminal voltage on shape of phase current at turn on angle $18 \mathrm{deg}$ and turn off angle $35 \mathrm{deg}$ at speed of $25 \mathrm{rad} / \mathrm{s}$.
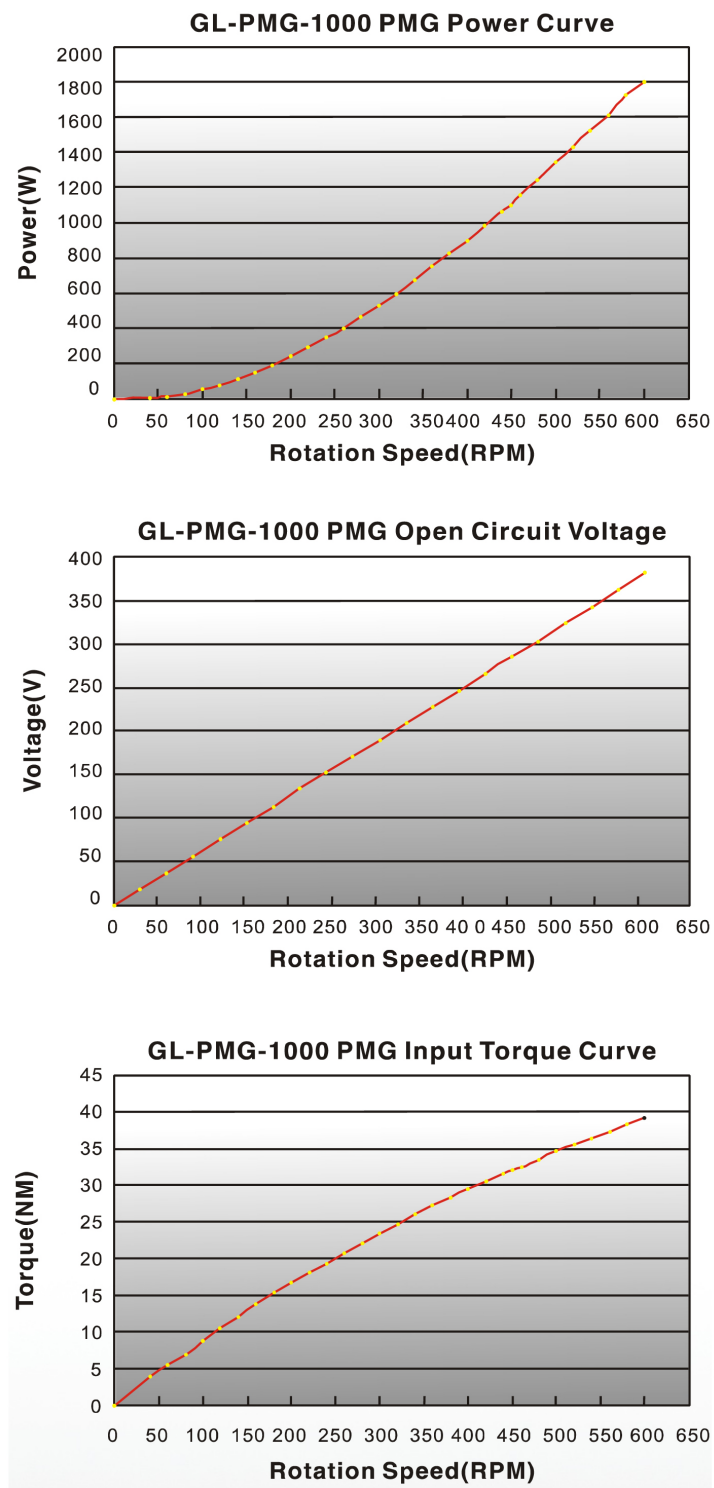

Figure 11. Generator characteristics. 


$$
T(\omega)=-0.0041 \omega^{2}+0.8788 \omega .
$$

The output voltage was not of concern to this project, as it is assumed that the downstream electrical systems will deal with that. Overall power generated is the important part. A MPPT (Maximum Power Point Tracking) controller may be required to maximize the output.

The generator was represented as a torque source in the simulation, taking the shaft speed as an input, and applying a torque to the system that opposed the direction of rotation.

\section{Simulation Results}

\subsection{Windmill}

In order to model the system as a whole, the windmill had to be modeled. This proved to be a less than straightforward affair because the torque generated by the turbine depended on both wind speed and the angular velocity of the rotor. The first model created (using the Simscape tools in the MATLAB and Simulink software packages), generated a very simple linear speed-torque characteristic. It did not take a direct wind speed input, instead using a stall torque. A rotational damper was used to make the torque reduce linearly as the rotor's angular velocity increased. In water pumping applications (where the American wind turbine is most commonly put to use) the starting torque is approximately 3 times that of the mean torque requirement when running [19]. This means the torque at the turbine's operating speed is one third of the starting torque.

While the simple, initial model produced a simple linear speed-torque curve, the reality is that it is a curve, albeit a fairly simple one [19]. As can be observed in Figure 2, the wind speed affects both speed and torque, and it appears that as wind speed increases, the curve is simply stretched in both speed and torque. This was an important assumption made in the model that was created.

The basic curve was recreated in Excel, and a regression applied to find a 3rd order polynomial fit. This equation did not directly take wind speed into account, instead working with a normalized rotor speed and torque. The modeled torque was then scaled up by an appropriate value, calculated from the rated speeds and torques for the designed turbine.

Prior to running the wind turbine model in the main combiner model, it was tested on its own to verify its accuracy. Figure 12 shows the varying speed torque curves for different wind speeds, as modeled. The small kinks at the bottom of the $1 \mathrm{~m} / \mathrm{s}, 3 \mathrm{~m} / \mathrm{s}$, and $5 \mathrm{~m} / \mathrm{s}$ curves are an artifact of the MATLAB simulation.

\subsection{Turgo Hydro Turbine}

A model for a Turgo turbine in the pico-hydro scale is generated in [20]. This model was implemented in Simulink and added to the already functioning model. No work was done in attempting to optimize those parameters for this project, except for the water head, jet diameter, and turbine radius. The model does not 
take into account windage losses inside the turbine housing. The parameters that were left as is include jet entry angle, water/turbine contact angles, and water exit angle. A $5 \mathrm{~cm}$ jet diameter was found to produce a flow of 19.7 litres/sec when fed with $5 \mathrm{~m}$ head water, matching my specifications of 20 litres/sec. The output of the model is illustrated in Figure 13, showing speed-torque curves for heads of 1 to 5 metres, in 1 metres increments.

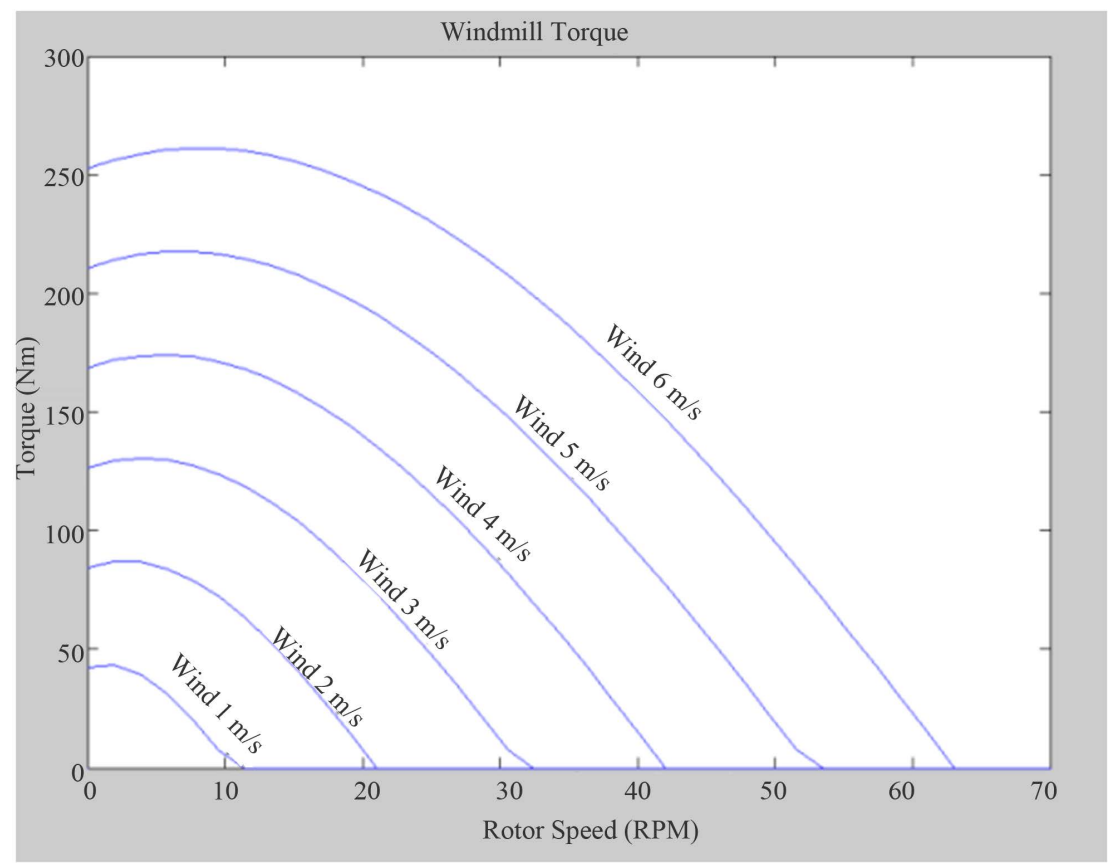

Figure 12. Wind turbine characteristics as modeled.

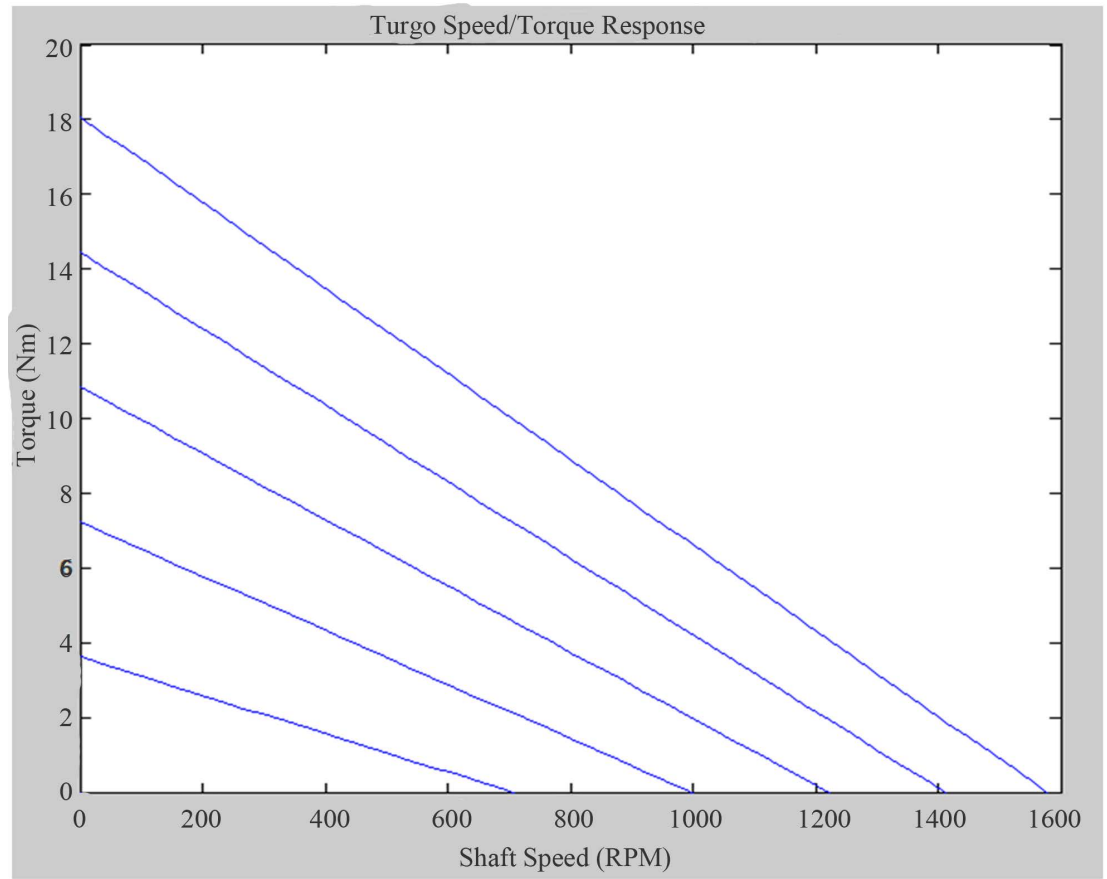

Figure 13. Turgo characteristics as modeled. 


\subsection{Combining System}

The "torque-split" combining system was modeled using the SimScape mechanical simulation toolbox, with the Simulink tool bundled with MATLAB. Only the fundamental mechanical blocks were used.

The initial modeling was attempted using first principles and a complete mathematical model of the system, using only the mathematical blocks in Simulink. However, this proved very difficult to debug and improve, as every fundamental property had to be incorporated into the model. For this reason the model was rewritten using the SimScape mechanical blocks, which took care of much of the underlying physics on their own, meaning it was much easier to concentrate efforts on the higher level parts of the system. The wind and hydro turbines were each modeled in their own custom code blocks, and each took an input of shaft speed and generated a simulated output torque from the model contained within. Figure 14 and Figure 15 show these custom code blocks within the main model.

The SimScape tools had common fundamental elements all ready to go, such as torque sources, inertia elements, gearboxes, sensors etc. The most significant inertia of the wind turbine, was found to be $120 \mathrm{~kg} \cdot \mathrm{m}^{2}$, by approximating the wind turbine as a disc, a valid assumption for the fairly solid American Wind pump.

A gearbox coupled each turbine to a common shaft that drove the generator. The generator itself was implemented such that it applied a torque opposing the

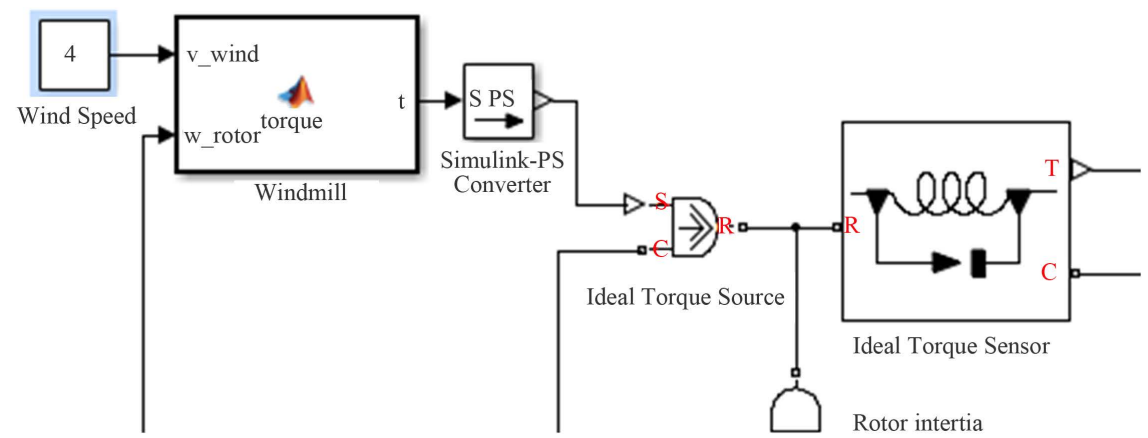

Figure 14. Wind turbine portion of main model.

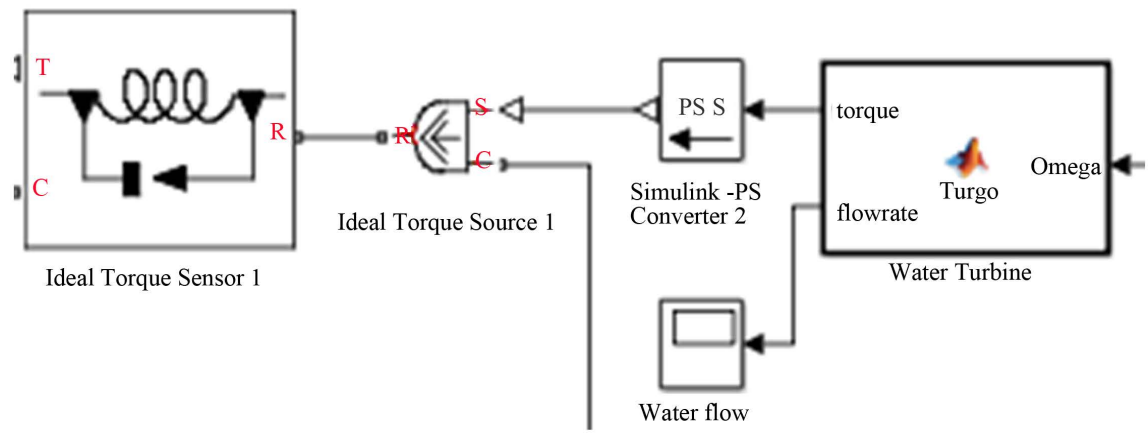

Figure 15. Hydro turbine portion of main model. 
direction of rotation of the common shaft. This torque was determined by the speed of the shaft. The gearbox ratios (1:12 step up for the wind turbine, 1:2 step down for the turgo) were determined by an iterative process of maximizing the power output for rated resource inputs $(6 \mathrm{~m} / \mathrm{s}$ wind, $201 / \mathrm{s}$ water @ $5 \mathrm{~m})$. In doing this, the power output was increased from 600 watts to 1040 watts.

\subsection{Results}

Graphing blocks were placed throughout the model to record what was happening within the system during simulation. The system settled at a steady state within a few seconds, after overcoming the inertias present. Figure 16 shows the power output of the generator over the first 10 seconds of operation. It settles at just below 1040 watts, using rated inputs.

Figure 17 shows the power output if the wind source is removed, leaving just the hydro turbine driving the generator. Note that it is assumed a freewheel clutch effectively removes the wind turbine from the system in such circumstances

Figure 18 shows the power output if the water turbine does not supply any power to the system, assuming a free wheel clutch is fitted on the output of the turgo turbine. The power output settles at 380 watts. This is of interest because it shows that the turbines, when driving together, are able to operate in a more efficient region, hence the generator is able to produce more power when both are driving together, than the sum of each turbine driving on its own.

\section{Analysis of Findings}

Renewable energy sources are intermittent, especially wind and solar. This

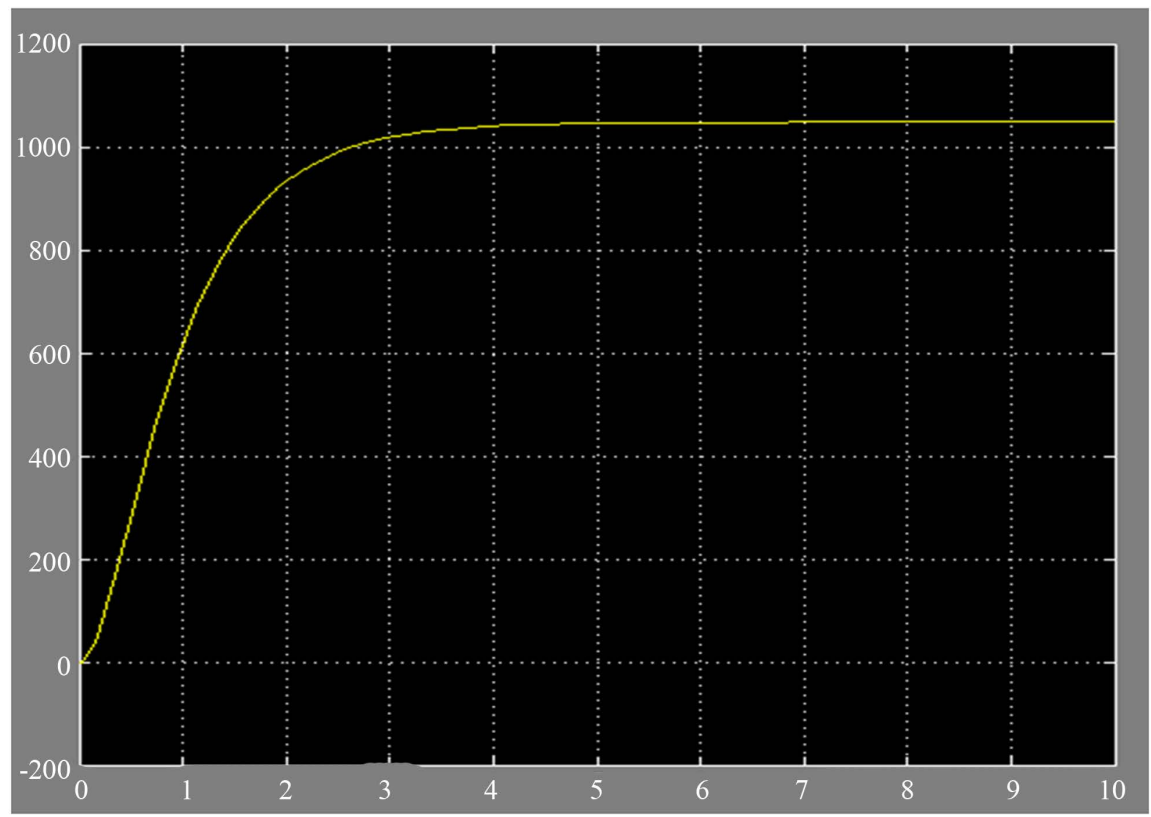

Figure 16. Power output over 10 seconds. 


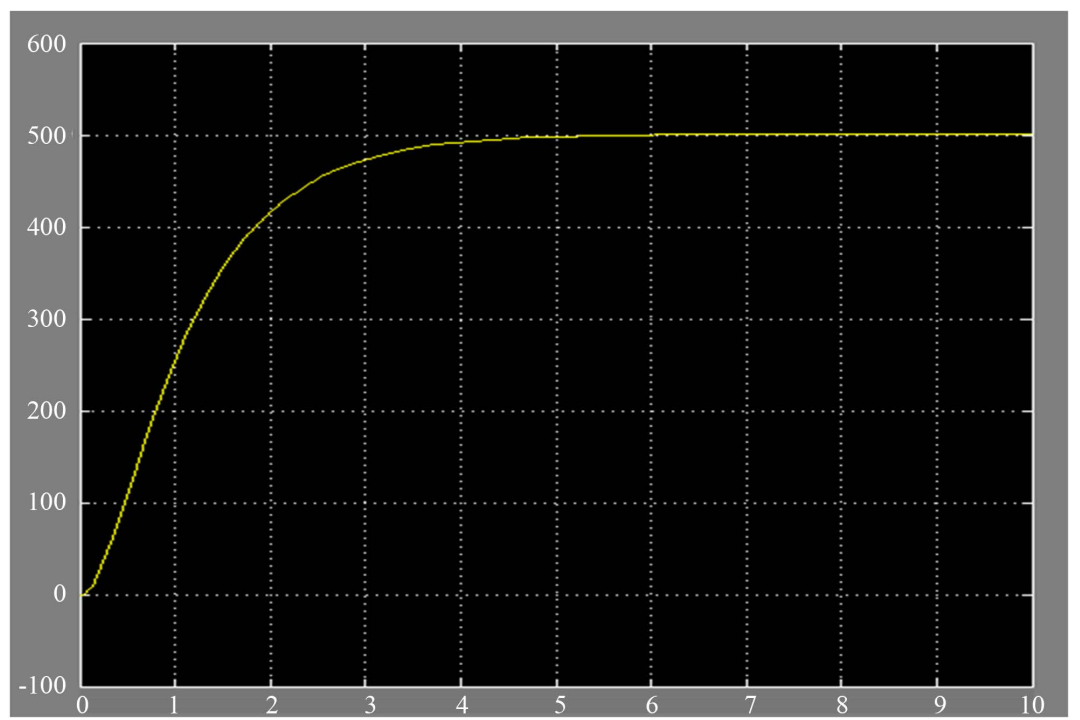

Figure 17. Power output with no wind.

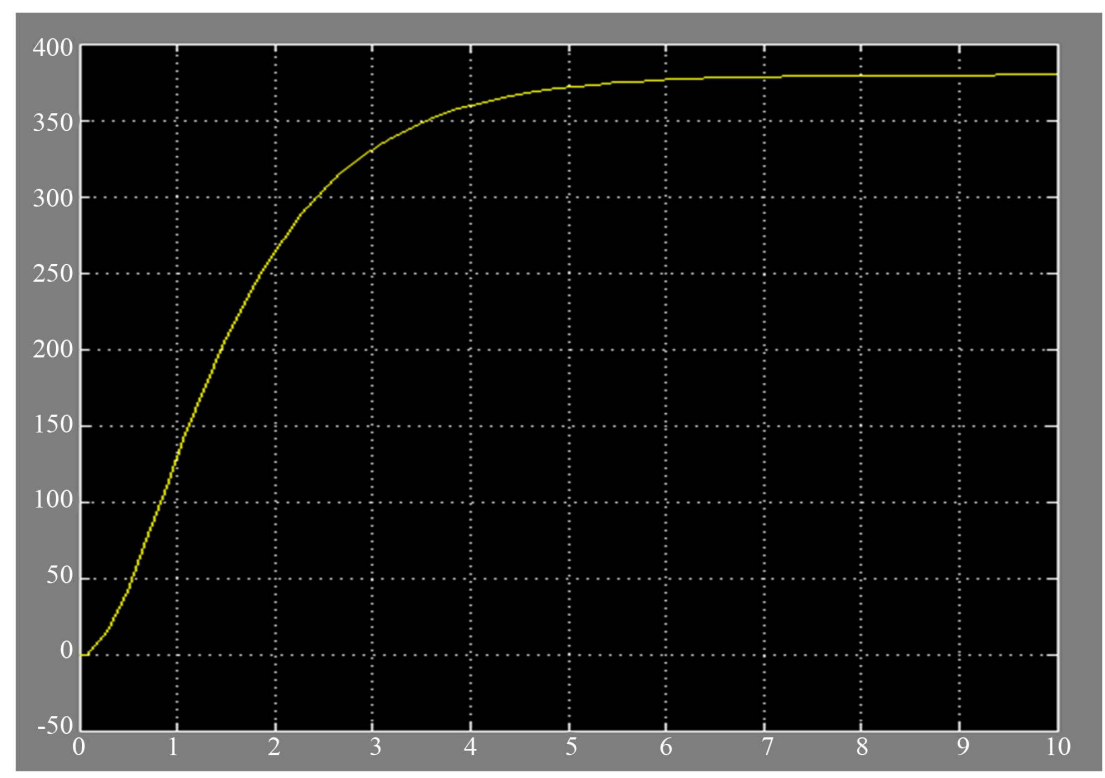

Figure 18. Power output using only wind, over first 10 seconds.

means that the system as designed will not produce its rated power continuously, but somewhat less. The average power output, long term, can be deduced by taking capacity factors into account for both the hydro and wind power sources. Conservative values for these were taken as 0.25 for the wind, and 0.9 for the hydro. In practice it is expected that the actual values for this are more like 0.35 and 0.99 , for a reliable, well designed, maintained, and situated system. Taking such conservative values allows for a simpler estimation to be done, using simple probability to calculate the long term output.

The average power output calculated in Table 3 translates to an annual generation capacity of over $5000 \mathrm{kWh}$. While less than the average usage, energy efficient houses and practices can allow a house to get by on this much power 
Table 3. Output power calculations.

\begin{tabular}{cccc}
\hline Power Source & Net Probability & Net Power & Net Power \\
\hline Wind + Hydro & $0.9^{\star} 0.25$ & $0.225^{\star} 1040$ & 234 \\
Wind & $0.1^{\star} 0.25$ & $0.025^{\star} 380$ & 9.5 \\
Hydro & $0.9^{\star} 0.75$ & $0.675^{\star} 500$ & 337.5 \\
None & $0.1^{\star} 0.75$ & $0.075^{\star} 0$ & 0 \\
& & Average Power & 581 Watts \\
\hline
\end{tabular}

without sacrificing very much. Of course, if combined with a grid-tie system, this is hardly a problem because power can simply be imported from the national grid as required.

\section{Conclusions}

This work is an attempt to make renewable energy more reliable, cost effective, and accessible by exploring a different energy combination system to that currently applied to wind and hydro power. Instead of the usual electrical combination of wind and hydro generators this work involved combining water and wind turbines mechanically, before driving an electrical generator. This new combination system is modeled and optimized in MATLAB, using a direct combination system commonly found in multi-engine helicopters. The system is found to operate satisfactorily. The research illustrates that there is potential for further research in the areas of pump-hydro storage and using a water pump as a turbine, both as an extension of this project. Research was undertaken regarding wind and water resource availability, and the turbines were chosen with these taken into consideration. Various combination systems were explored, including torque and speed split mechanical combinations, conventional electrical combination, and using a modified switched reluctance generator as a method of electro-mechanical combination. The system met the requirements in terms of resource constraints and power output. The new combination method was modeled in MATLAB, taking advantage of previous work done on Turgo modeling and the results obtained are highly encouraging to conduct the further research in the area.

A three phase 12/8 Switched Reluctance (SR) machine is used as a generator for the study. A detailed winding polarity having four poles per phase and their effect on the performance of the machine is observed. From the collected data, the optimal firing angles that yield the highest percentage of generated power at each different speed and voltage level were identified.

\section{Acknowledgements}

The author wishes to thank the students and the workshop-technical staff at Massey University-School of Engineering and Advanced Technology for their help and support in this work. 


\section{References}

[1] Spertino, F., Ciocia, A., Cocina, V. and Di Leo, P. (2016) Renewable Sources with Storage for Cost-Effective Solutions to Supply Commercial Loads. 2016 International Symposium on Power Electronics, Electrical Drives, Automation and Motion, Anacapri, 22-24 June 2016, 242-247. https://doi.org/10.1109/SPEEDAM.2016.7525987

[2] Saheb-Koussa, D., Koussa, M.S., Bellarbi, M. and Boufertella, A. (2016) Fuzzy Logic Management Supervisor for Wind-Diesel-Battery Hybrid Energy System. 20167 th International Renewable Energy Congress, Hammamet, 22-24 March 2016, 1-6. https://doi.org/10.1109/IREC.2016.7478947

[3] Zhou, T. and François, B. (2011) Energy Management and Power Control of a Hybrid Active Wind Generator for Distributed Power Generation and Grid Integration. IEEE Transactions on Industrial Electronics, 58, 95-104. https://doi.org/10.1109/TIE.2010.2046580

[4] Huang, R., Low, S.H., Topcu, U., Chandy, K.M. and Clarke, C.R. (2011) Optimal Design of Hybrid Energy System with PV/Wind Turbine/Storage: A Case Study. 2011 IEEE International Conference on Smart Grid Communications, Brussels, 17-20 October 2011, 511-516. https://doi.org/10.1109/SmartGridComm.2011.6102376

[5] Santarelli, M., Cali, M. and Macagno, S. (2004) Design and Analysis of Stand-Alone Hydrogen Energy Systems with Different Renewable Sources. International Journal of Hydrogen Energy, 29, 1571-1586. https://doi.org/10.1016/j.ijhydene.2004.01.014

[6] Deshmukh, M.K. and Deshmukh, S.S. (2008) Modeling of Hybrid Renewable Energy Systems. Renewable and Sustainable Energy Reviews, 12, 235-249.

https://doi.org/10.1016/j.rser.2006.07.011

[7] Tomislav, D., Lu, X., Vasquez, J.C. and Josep, M. (2016) DC Microgrids-Part II: A Review of Power Architectures, Applications and Standardization Issues. IEEE Transactions on Power Electronics, 31, 3528-3549.

https://doi.org/10.1109/TPEL.2015.2464277

[8] Mira, M.C., Zhang, Z., Knott, A. and Andersen, M.A.E. (2016) Analysis, Design, Modelling and Control of an Interleaved-Boost Full-Bridge Three-Port Converter for Hybrid Renewable Energy Systems. IEEE Transactions on Power Electronics, 32, 1138-1155. https://doi.org/10.1109/TPEL.2016.2549015

[9] Moury, S., Lam, J., Srivastava, V. and Church, R. (2016) A Novel Multi-Input Converter Using Soft-Switched Single-Switch Input Modules with Integrated Power Factor Correction Capability for Hybrid Renewable Energy Systems. 2016 IEEE Applied Power Electronics Conference and Exposition, Long Beach, 20-24 March 2016, 786-793. https://doi.org/10.1109/APEC.2016.7467961

[10] Jiang, W. and Fahimi, B. (2009) Multi-Port Power Electric Interface for Renewable Energy Sources. 2009 Twenty-Fourth Annual IEEE Applied Power Electronics Conference and Exposition, Washington DC, 15-19 February 2009, 347-352. https://doi.org/10.1109/APEC.2009.4802680

[11] Rehman, Z., Al-Bahadly, I. and Mukhopadhyay, S. (2015) Dual Input-Dual Output Single Inductor DC-DC Converter for Renewable Energy Applications. International Conference on Renewable Energy Research and Applications, Palermo, 22-25 November 2015, 783-788.

[12] Schofield, N. and Long, S. (2009) Generator Operation of a Switched Reluctance Starter/Generator at Extended Speeds. IEEE Transactions on Vehicular Technology, 58, 48-56. https://doi.org/10.1109/TVT.2008.924981 
[13] Jayapragash, R. and Chellamuthu, C. (2013) Analysis of Switched Reluctance Machine using FEA for Renewable Energy System. International Conference on Power, Energy and Control, Sri Rangalatchum Dindigul, 6-8 February 2013, 777-781. https://doi.org/10.1109/ICPEC.2013.6527760

[14] Kioskeridis, I. and Mademlis, C. (2006) Optimal Efficiency Control of Switched Reluctance Generators. IEEE Transactions on Power Electronics, 21, 1062-1072. https://doi.org/10.1109/TPEL.2006.876827

[15] Mademlis, C. and Kioskeridis, I. (2005) Optimizing Performance in Current-Controlled Switched Reluctance Generators. IEEE Transactions on Energy Conversion, 20, 556-565. https://doi.org/10.1109/TEC.2005.852960

[16] Cardenas, R., Pena, R., Perez, M., Clare, J., Asher, G. and Wheeler, P. (2005) Control of a Switched Reluctance Generator for Variable-Speed Wind Energy Applications. IEEE transactions on Energy conversion, 20, 781-791. https://doi.org/10.1109/TEC.2005.853733

[17] Chen, H. and Shao, Z. (2004) Turn-On Angle Control for Switched Reluctance Wind Power Generator System. 30th Annual Conference of IEEE Industrial Electronics Society, Busan, 2-6 November 2004, 2367-2370.

https://doi.org/10.1109/IECON.2004.1432170

[18] NIWA (n.d.) Wind Energy Map.

https://www.niwa.co.nz/sites/niwa.co.nz/files/imported/_data/assets/image/0004/5 0539/renewable3_large.gif

[19] Fraenkel, P., Barlow, R., Crick, F., Derrick, A. and Bokalders, V. (1993) Wind Pumps, A Guide for Development Workers. Intermediate Technology Publications Ltd., Nottingham. https://doi.org/10.3362/9781780446448

[20] Williamson, S.J., Stark, B.H. and Booker, J.D. (2013) Performance of a Low-Head Picohydro Turgo Turbine. Applied Energy, 102, 1114-1126. https://doi.org/10.1016/j.apenergy.2012.06.029

[21] Miller, J.M. (2006) Hybrid Electric Vehicle Propulsion System Architectures of the e-CVT Type. IEEE Transactions on Power Electronics, 21, 756-767. https://doi.org/10.1109/TPEL.2006.872372 